\title{
MODELLING OF SPRAYS USING COMPUTATIONAL FLUID DYNAMICS CODES
}

\author{
Sergei Sazhin \\ Sir Harry Ricardo Laboratory, School of Environment and Technology, \\ University of Brighton, Brighton BN2 4GJ, UK, e-mail: S.Sazhin@ brighton.ac.uk
}

Received 2 January 2009; accepted ???? 2009

\begin{abstract}
Recently developed approaches to droplet and spray modelling in computational fluid dynamics (CFD) codes are reviewed. It is emphasized that the modelling of spray primary break-up needs to take into account the transient nature of sprays, while the modelling of droplet heating and evaporation needs to take into account a number of factors, including recirculation and finite thermal conductivity of liquid, semi-transparency of droplets and the kinetic effects. This modelling needs to take place in realistic three-dimensional enclosures, which makes it essential to find a compromise between the accuracy of the models and their CPU efficiency.
\end{abstract}

Keywords: Sprays, Droplets, Computational Fluid Dynamics Codes, Break-up, Heating, Evaporation

\section{Introduction}

The importance of the accurate multi-dimensional modelling of droplets and sprays | in various engineering and environmental applications is well recognised (e.g. [1]-[4]). In the most general case, the models need to take into account a number of complicated fluid dynamics, heat/mass transfer and combustion processes in realistic threedimensional configurations. This inevitably leads to the application of rather simplistic models describing individual processes. These include simplistic chemical models, droplet break-up models, which do not take into account transient effects, and droplet heating models, which disregard the effects of internal temperature gradient, and so on. The aim of this paper is to summarise the results of recent studies into the necessity for, and feasibility of, relaxing some of the simplifying assumptions in the modelling of droplet break-up, heating and evaporation in engineering computational fluid dynamics (CFD) codes. Some of the new models have been implemented into the customised 
version of the KIVA 2 computational fluid dynamics (CFD) code. The implementation of these models into other CFD codes seems to be a straightforward task.

The modelling of the gas phase in CFD codes is generally based on the Eulerian approach when the analysis is focused on the solution of discretised equations of conservation of mass, momentum and energy for specific computational cells [5, 6]. An alternative approach is based on the solution of the vorticity equation [7]-[10], but this is rarely used in practical engineering applications. As for spray modelling, two approaches have been developed and used: the Eulerian approach, when liquid is treated as a continuous medium or as the second phase of the multiphase flow (e.g. [11]), and the Lagrangian approach when the analysis is focused on tracing the trajectories of individual droplets or groups of droplets (droplet parcels) on a sub-grid scale (e.g. [12]). The second approach is generally more popular than the Eulerian one [13]. It is implemented in an open-source non-commercial computer code KIVA, widely used for development and validation of spray models $[14,15]$.

The Lagrangian approach to spray modelling has a number of well-known limitations, including the grid dependency of the results for dense sprays. These limitations and the ways in which they can be overcome were discussed in [16]. One of the most important elements of Lagrangian spray models is their focus on jet and droplet break-up. The nature of the break-up process depends on a spray region. Primary break-up takes place near the nozzle exit. Here disintegration of the liquid jet occurs. In the far-field spray, where the liquid phase is dispersed in the gas, the secondary break-up of large droplets into smaller ones takes place. In many practical applications, unified models have been used for both primary and secondary break-up. In these models, the jet is approximated by a chain of droplets, with initial diameters equal to the diameter of the nozzle, or slightly less than this diameter if the effects of cavitation are taken into account [17]. For implementation into CFD codes, designed for computation of three-dimensional sprays, the so-called TAB (Taylor Analogy Break-up) and WAVE models of break-up and their modifications are commonly applied [18]. An important limitation of the above-mentioned break-up models relates to the fact that they are based on an unrealistic assumption about single-size droplets created after the break-up. More realistic stochastic models describe the break-up in terms of the evolution of the droplet distribution function over time ([19]-[23]). Another limitation of the break-up models used in CFD codes stems from an assumption about quasi-steady-state flow conditions, although in many practical applications spray injection is an essentially transient process.

As for the modelling of heating and evaporation of individual droplets, only the simplest models have been implemented into most available CFD codes. These models are based on the assumption that the thermal conductivity of droplets is infinitely high so that there are no temperature gradients inside them. In evaporation models it has been assumed that vapour in the vicinity of a droplet's surface is always saturated, and so the problem of droplet evaporation reduces to the problem of vapour diffusion to the ambient gas (these are the hydrodynamic models) [4].

In what follows, attempts to overcome these limitations of the model in CFD codes will be described. 


\section{Break-up of transient sprays}

In order to account for the effect of transient injection on break-up, the analysis was focused on the WAVE model, the parameters of which, controlling the rate of spray disintegration, have been modified [24]. The original WAVE break-up model is based on the analysis of the Kelvin-Helmholtz instability of a liquid jet. This instability leads to stripping of child droplets from the liquid core. The core is approximated by parent droplet parcels injected from the nozzle. The radii of the droplets in these parcels $R_{d}$ continuously decrease during the break-up process, as described by the following equation:

$$
\frac{d R_{d}}{d t}=-\frac{R_{d}-R_{e q}}{\tau_{b u}}
$$

where $\tau_{b u}$ is the characteristic break-up time, and $R_{e q}$ is the radius of droplets in equilibrium (stable droplets):

$$
R_{e q}=\left\{\begin{array}{cc}
B_{o} \Lambda, & B_{o} \Lambda \leq R_{d} \\
\min \left(\begin{array}{c}
\left(3 \pi R^{2} U / 2 \Omega\right)^{0.33} \\
\left(3 R^{2} \Lambda / 4\right)^{0.33}
\end{array}\right), & B_{o} \Lambda>R_{d}
\end{array}\right.
$$

$B_{o}=0.61$ is the model constant, $\Lambda$ and $\Omega$ are the wave length and the frequency of the fastest growing disturbance on the surface of a liquid jet moving in an inviscid gas. These are functions of Reynolds, Weber and Ohnesorge numbers [25]. The break-up time $\tau_{b u}$ is estimated as:

$$
\tau_{b u}=3.726 \cdot B_{1} \frac{R_{d}}{\Lambda \Omega} .
$$

The break-up constant $B_{1}$ was taken to be equal to 10 [25] based on the results of measurements of quasi-steady-state Diesel spray penetration at relatively low injection rates. Later, it was shown that the break-up constant $B_{1}$ can vary widely depending on the type of injector.

Patterson and Reitz [26] developed this model further in order to account for the effect of the Rayleigh-Taylor (RT) instability of droplets. When the wave length corresponding to the maximal increment of this instability

$$
\Lambda_{R T}=2 \pi \sqrt{\frac{3 \sigma}{a \rho_{l}}}
$$

is less than the diameter of a droplet, the bag break-up of the droplet is expected to take place. In this expression, $\sigma$ is the surface tension, and $a=\frac{3}{4} C_{D} \frac{\rho_{g} U^{2}}{\rho_{l} D}$ is the deceleration of the droplet due to the drag force. For the Rayleigh-Taylor type of break- 
up, $R_{e q}$ was calculated as $R_{e q}=C_{R T} \Lambda_{R T}$, where $C_{R T}=2.5$ is the model constant [26]. The break-up time was estimated as:

$$
\tau_{b u, R T}=\sqrt{\frac{3}{2 a} \sqrt{\frac{3 \sigma}{a \rho_{l}}}} .
$$

The Kelvin-Helmholtz instability is mainly responsible for the primary break-up, while the Rayleigh-Taylor mechanism prevails at the secondary atomisation stage. In both cases the droplets conserve momenta during the break-up process.

In the modified version of the WAVE model, the decrease in $\Omega$ (the frequency of the fastest growing disturbance on the liquid surface) with increasing injection acceleration was taken into account, while it was assumed that the wavelength of critical instability $\Lambda$ is not affected by the transient nature of the flow. This decrease is accounted for by introducing the following relation for $B_{1}$ :

$$
B_{1}=B_{1, s t}\left[1+c_{1} \cdot\left(a^{+}\right)^{c_{2}}\right]
$$

where $a^{+}=\sqrt{\operatorname{Re}} \frac{D}{U_{i n j}^{2}} \cdot \frac{d U_{i n j}}{d t}$ is the acceleration parameter taking into account the effect of flow acceleration; $c_{1}$ and $c_{2}$ are adjustable constants. In the steady-state limit, $a^{+}$is zero and $B_{1}=B_{1, s t}$. Following [25], it was assumed that $B_{1, s t}=10$.

A rigid body concept to describe the propagation of disturbances along the liquid core was introduced. It was assumed that parcels constituting the liquid core experience no drag from the gas and move as a rigid jet at a speed equal to the instantaneous injection velocity $U_{j e t}=U_{i n j}$. The stripping of the jet due to the development of the KelvinHelmholtz instability, however, is allowed. This leads to a continuous decrease in the diameter $D$ of parcels in the spray core described by Equation (1). In computations, the length of the liquid core has been limited by the condition $D<0.5 D_{i n j}$, similar to the criterion applied by Reitz [25] ( $D_{i n j}$ is the initial jet diameter at the nozzle exit). The introduction of the critical diameter of the liquid core allows us to distinguish between the primary and the secondary break-up regions in the model.

The results of calculations of spray tip penetration, using the conventional TAB and WAVE (with $B_{l}=10$ ), modified WAVE and stochastic (suggested in [19]) models, and the corresponding experimental data for a highly transient spray, were compared [24]. As follows from this comparison, the conventional WAVE model, TAB and stochastic models significantly under-predict the penetration at the initial stage of this process. At the same time the modified version of the WAVE model, described above, gave much better agreement between the predictions of the model and experimental data, as expected, remembering the highly transient nature of the spray under consideration [27]. It is recommended that this model is implemented into other CFD codes used for the analysis of transient sprays. 


\section{Modelling of droplet heating and evaporation}

Although the heating and evaporation of droplets is a complex process, involving a close interaction between the liquid and gas phases (e.g. [28]), the modelling of these processes in CFD codes is commonly based on the separate analysis of the two phases.

\subsection{Liquid phase models}

The liquid phase models actually used in CFD codes, or the ones which can potentially be used, are the ITC (infinite thermal conductivity) and ETC (effective thermal conductivity) models. The ITC models are based on the energy balance equation of the droplet as a whole. The solution to this equation can be presented as [1, 4]:

$T=T_{s}=T_{g}+\left(T_{s 0}-T_{g}\right) \exp \left(-\frac{3 h t}{c_{l} \rho_{l} R_{d}}\right)$,

where $T_{s 0}$ and $T_{g}$ are the initial droplet temperature and ambient gas temperature

respectively, $c_{l}$ and $\rho_{l}$ are liquid specific heat capacity and density respectively. Droplet temperature $T$ does not depend on the distance from the droplet centre $R$ in this case.

Assuming that the process is spherically symmetrical, the droplet transient heating in finite liquid thermal conductivity models is described by the following equation [4]:

$\frac{\partial T}{\partial t}=\frac{K_{l}}{R^{2}} \frac{\partial}{\partial R}\left(R^{2} \frac{\partial T}{\partial R}\right)+P(t, R)$,

where $K_{l}=k_{l} /\left(c_{l} \rho_{l}\right)$ is the liquid thermal diffusivity, $k_{l}$ is the liquid thermal conductivity, assumed to be constant, $T$ is specified at the initial moment of time as $\left.T\right|_{t=0}=T_{0}(R), P$ takes into account the radiative heating of a droplet. The boundary condition at $R=0$ follows from the problem symmetry $\partial T /\left.\partial R\right|_{R=0}=0$. It was assumed that the droplet is heated by convection from the surrounding gas, and cooled due to evaporation. The general analytical solution of Equation (8), taking into account the changes in droplet radius due to evaporation, would be a difficult task. This could be considerably simplified if we take into account that this solution is used in the numerical analysis when the time step is small. In this case we can assume that the droplet radius is constant, but the effect of evaporation can be taken into account by replacing $T_{g}$ with the effective temperature [4]:

$$
T_{\text {eff }}=T_{g}+\rho_{l} L \frac{d R_{d}}{d t} / h
$$

where $L$ is the latent heat of evaporation, effects of swelling are ignored at this stage, $h$ is the convection heat transfer coefficient. The solution to (8) for $h=$ const can be presented as [4]: 
$T=T_{e f f}+\frac{R_{d}}{R} \sum_{n=1}^{\infty}\left\{\left[q_{n}-\frac{\sin \lambda_{n} \mu_{0}(0)}{\lambda_{n}^{2}\left\|V_{n}\right\|^{2}}\right] \exp \left(-\kappa_{0} \lambda_{n}^{2} t\right)\right.$

$\left.-\frac{\sin \lambda_{n}}{\lambda_{n}^{2}\left\|V_{n}\right\|^{2}} \int_{0}^{1} \frac{d \mu_{0}(\tau)}{d \tau} \exp \left[-\kappa_{0} \lambda_{n}^{2}(t-\tau)\right] d \tau\right\}$

$\times \sin \left(\frac{\lambda_{n} R}{R_{d}}\right)+\frac{R_{d}}{R} \sum_{n=1}^{\infty} \frac{p_{n}}{\kappa_{0} \lambda_{n}^{2}}\left[1-\exp \left(-\kappa_{0} \lambda_{n}^{2} t\right)\right] \sin \left(\frac{\lambda_{n} R}{R_{d}}\right)$

where

$\mu_{0}=\frac{h T_{e f f}(t) R_{d}}{k_{l}}$,

$q_{n}=\frac{1}{\left\|V_{n}\right\|^{2}} \int_{0}^{1} \bar{T} V_{n}(R) d\left(R / R_{d}\right)$,

$T_{d 0}$ is the droplet initial temperature distribution,

$\bar{T}=R T_{d 0}(R) / R_{d}, \kappa_{0}=k_{l} /\left(c_{l} \rho_{l} R_{d}^{2}\right), \lambda_{n}$ are solutions to the equation:

$\lambda_{n} \cos \lambda_{n}+h_{0} \sin \lambda_{n}=0$,

$h_{0}=\frac{h r_{d}}{k_{l}}-1,\left\|V_{n}\right\|^{2}=0.5\left(1-\sin 2 \lambda_{n} / 2 \lambda_{n}\right)$,

$V_{n}=\sin \left(\lambda_{n} R / R_{d}\right)$,

$p_{n}=\frac{1}{\left\|V_{n}\right\|^{2}} \int_{0}^{1}\left[\frac{R P(t, R)}{R_{d}}\right] V_{n}(R) d\left(R / R_{d}\right)$,

$P(R)$ takes into account the radiative heating of droplets. The variations of all parameters with temperature and time were accounted for when analytical solution (10) was incorporated into a numerical code.

The finite liquid thermal conductivity model could be generalised to take into account the internal recirculation inside droplets. This could be achieved by replacing the thermal conductivity of liquid $k_{l}$ with the so-called effective thermal conductivity $k_{\text {eff }}=\chi$ $k_{l}$, where the coefficient $\chi$ varies from about 1 (at droplet Peclet number $<10$ ) to 2.72 (at droplet Peclet number >500) [29]. This is known as the effective thermal conductivity (ETC) model.

Various models for $P(R)$, taking into account the variation of thermal radiation absorption inside droplets were suggested (see [4]). It transpired that this function has only minor effects on the heating of droplets, which can be ignored in most practical applications. Ignoring the dependence of the distribution of thermal radiation absorption on $R$, we can present the expression for $P(R)$ as [4]: 
$P(R)=\frac{3 \sigma_{S B}}{\rho_{l} c_{l}} a R_{d}^{b-1} T_{e x t}^{4}$,

where we assume that the radiation temperature $\theta_{R}$ is equal to the external temperature $T_{\text {ext }}, a$ and $b$ are polynomials (quadratic functions in most cases) of $\theta_{R}, \sigma_{S B}$ is the StefanBoltzmann constant. For low sulphur ESSO AF 1313 Diesel fuel, it was found that the best approximation for $a$ and $b$ in the ranges $5 \mu \mathrm{m} \leq R_{d} \leq 50 \mu \mathrm{m}$ and $1000 \leq T_{\text {ext }} \leq 3000 \mathrm{~K}$ is provided by the functions [4]:

$a=0.10400-0.05432\left(T_{\text {ext }} / 1000\right)+0.00800\left(T_{\text {ext }} / 1000\right)^{2}$.

$b=0.49162-0.09837\left(T_{\text {ext }} / 1000\right)-0.007857\left(T_{\text {ext }} / 1000\right)^{2}$.

Equation (16) is essentially an approximation of the prediction of the Mie solution for the absorption of thermal radiation by semi-transparent droplets. Note that in all CFD codes known to us the effects of the semi-transparency of droplets are not taken into account and the droplets are treated as grey opaque spheres (see [4] for a more detailed analysis of this problem).

\subsection{Gas phase hydrodynamic models}

The values of $h$ are controlled by the conditions in the gas phase. Various approximations for $h$ are usually described in terms of the corresponding approximations for the Nusselt number $\mathrm{Nu}=h R_{d} / k_{g}$. Droplet heating, discussed in the previous section, is accompanied by droplet evaporation, which is described by the following equation:

$\frac{d R_{d}}{d t}=-\frac{k_{g} \mathrm{Sh}}{2 \rho_{l} c_{p g} R_{d}}$,

where $k_{g}$ is the gas thermal conductivity, $c_{p g}$ is the gas specific heat capacity at constant pressure, Sh is the Sherwood number. The difference between various gas models is essentially described in terms of the approximations of Nusselt and Sherwood numbers [30]. As shown in [30], the gas phase model, originally suggested in [29], predicts the evaporation time closest to the one based on the approximation of experimental data. This gas phase model was recommended for practical application in CFD codes. In most cases, the droplet evaporation time depends strongly on the choice of gas phase model. The dependence of this time on the choice of liquid phase model, however, is weak if the droplet break-up processes are not taken into account. On the other hand, the dependence of the droplet surface temperature, at the initial stage of heating and evaporation, on the choice of gas phase model is weak, while its dependence on the choice of liquid phase model is strong [30].

Solution (10) with the radiation term in the form (16) was used in the new approach to the numerical modelling of droplet heating and evaporation by convection and radiation from the surrounding gas [4]. This solution was applied at the first time step, using the initial distribution of temperature inside the droplet. The results of the analytical solution over this time step were used as the initial condition for the second 
time step, and so on. This approach was compared with those approaches based on the numerical solution of the discretised heat conduction Equation (8), and those based on the assumption that there is no temperature gradient inside the droplets (see Equation (7)) [4]. All of these approaches were applied to the numerical modelling of fuel droplet heating and evaporation in conditions relevant to Diesel engines, but without taking into account the effects of droplet break-up. The algorithm based on the analytical solution for constant $h$ has been shown to be more effective (from the points of view of accuracy and CPU time requirement) than the approach based on the numerical solution of the discretised heat conduction equation inside the droplet, and more accurate than the solution ignoring the temperature gradient inside droplets [4]. This numerical algorithm was implemented into a zero-dimensional code, used for the modelling of coupling between droplets and gas, and the KIVA II CFD code [24]. The importance of taking into account the effects of temperature gradients inside droplets was confirmed by direct temperature measurements inside droplets [31]. The validity of the effective thermal conductivity model with the simplified radiation term was justified by direct comparison of the prediction of this model and the rigorous model, taking into account the recirculation inside, and the distribution of the radiation absorption inside, droplets $[32,33]$. It was recommended that the effects of temperature gradient inside droplets are taken into account in computational fluid dynamics codes, using the model described above.

\subsection{Gas phase kinetic models}

As mentioned in the introduction, the currently used gas phase hydrodynamics models for droplet evaporation, including the one described in the previous section, implicitly assume that the rate of detachment of molecules of fuel is such that the concentration of fuel vapour at the droplet surface is maintained at saturation level. The applicability of this assumption to the problem of modelling Diesel fuel droplet evaporation in realistic Diesel engines is not at first evident. In [34] a comparative analysis of hydrodynamic and kinetic approaches to the problem of Diesel fuel droplet evaporation was presented, based on a previously developed approximate kinetic model. This model was based on a number of assumptions the applicability of which to realistic (including Diesel engine) conditions is not immediately obvious. For example the contribution of air in the vicinity of the droplet surfaces was ignored.

A new numerical kinetic model for droplet evaporation into a high-pressure background gas, approximated by air, is described in [35]-[37]. As in [32], two regions above the surface of the evaporating droplet were considered. These are the kinetic region, where the analysis is based on the Boltzmann equation, and the hydrodynamic region. The contribution of air and heat flux in the kinetic region was taken into account.

The evolution of the distribution function of air $f_{a} \equiv f_{a}(\mathbf{r}, \mathbf{v}, t)$ and fuel vapour $f_{v} \equiv f_{v}(\mathbf{r}, \mathbf{v}, t)$ in the kinetic region is controlled by the corresponding Boltzmann equations: 
$\frac{\partial f_{a}}{\partial t}+\mathbf{v}_{a} \frac{\partial f_{a}}{\partial \mathbf{r}}=J_{a a}+J_{a v}$

$\frac{\partial f_{v}}{\partial t}+\mathbf{v}_{v} \frac{\partial f_{v}}{\partial \mathbf{r}}=J_{v a}+J_{v v}$

where $J_{\alpha \beta}(\alpha=a, v ; \beta=a, v)$ are collision integrals defined as

$J_{\alpha \beta}=\frac{\sigma_{\alpha \beta}^{2}}{2} \int_{-\infty}^{+\infty} \mathrm{d} \mathbf{v}_{1} \int_{0}^{\pi} \sin \theta \mathrm{d} \theta \int_{0}^{2 \pi} \mathrm{d} \phi\left(f_{\alpha}^{\prime} f_{\beta 1}^{\prime}-f_{\alpha} f_{\beta 1}\right)\left|\mathbf{v}_{\alpha}-\mathbf{v}_{\beta 1}\right|$,

$\sigma_{\alpha \beta}=\left(\sigma_{\alpha}+\sigma_{\beta}\right) / 2, \sigma_{\alpha}$ and $\sigma_{\beta}$ are the corresponding diameters of molecules, $\theta$ and $\phi$ are angular coordinates of molecules $\beta$ relative to molecules $\alpha$ after the collision, superscript ' indicates the velocities and the distribution functions after collisions, subscript ${ }_{1}$ indicates that molecules of type $\beta$ collide with molecules of type $\alpha$ and as a result of this interaction the function $f_{\alpha}$ is modified. When deriving these equations it was assumed that molecules are rigid elastic spheres and body forces acting on them are negligible. A modified version of the previously developed method of direct numerical solution of these Boltzmann equations was used.

Although the model described in [35]-[37] shows considerable progress in the development of kinetic models for droplet evaporation, it still has a number of important limitations. Its predictions still rely heavily on the choice of the value of the evaporation coefficient. The difficulties with the measurement or calculation of this coefficient are well known. In most cases it can be assumed that the system is in a state of equilibrium, when the evaporation coefficient coincides with the condensation coefficient. The rigorous theoretical estimation of both coefficients would require the application of molecular dynamics methods, the analysis of which is beyond the scope of this paper (see [38-40]). Also, it remains unclear how kinetic effects can possibly be taken into account in CFD codes.

In the most recent developments the kinetic effects were described as perturbations of the predictions of the hydrodynamic models, using simple approximations [41, 42]. This | approach is expected to open the way to taking- kinetic effects into account in CFD codes. The work in this direction has not yet been completed.

\section{Conclusion}

Recently developed models for transient spray break-up, droplet heating and evaporation, suitable for implementation into computational fluid dynamics (CFD) codes, are reviewed with a view to specific application to the modelling of the processes in Diesel engines. It is pointed out that the gas phase model, taking into account the finite thickness of the thermal boundary layer around the droplet, in the form suggested by Abramzon and Sirignano [29], predicts the evaporation time closest to the one based on the approximation of experimental data. This gas phase model is recommended for 
practical application in CFD codes. In most cases, the droplet evaporation time depends strongly on the choice of the gas phase model. The dependence of this time on the choice of the liquid phase model, however, is weak if the droplet break-up processes are not taken into account. On the other hand, the dependence of the droplet surface temperature, at the initial stage of heating and evaporation, on the choice of the gas phase model is weak, while its dependence on the choice of the liquid phase model is strong. The relatively small contribution of thermal radiation to droplet heating and evaporation allows us to describe it using a simplified model, which takes into account their semi-transparency, but does not consider the spatial variations of radiation absorption inside droplets. The algorithm based on the analytical solution for constant $h$ is more effective (from the points of view of accuracy and CPU time requirement) than the approach based on the numerical solution of the discretised heat conduction equation inside the droplet, and more accurate than the solution based on the assumption that the thermal conductivity inside droplets is infinitely large. It is recommended that kinetic effects are taken into account when accurate analysis of Diesel fuel droplet evaporation is essential. They may be taken into account in CFD codes if the results of kinetic calculations are described as the perturbations to the predictions of the hydrodynamic models, using simple approximations.

\section{Acknowledgements}

The author is grateful to the Royal Society for the financial support of this project.

\section{References}

[1] Sirignano WA. Fluid Dynamics and Transport of Droplets and Sprays, Cambridge, UK: Cambridge University Press, 1999.

[2] Michaelides EE. Particles, Bubbles \& Drops. New Jersey: World Scientific, 2006.

[3] Jancskar I, Ivanyi A Phenomenological hysteresis model for vapour-liquid phase transition, Pollack Periodica, Vol. 3,2008, pp. 5-28.

[4] Sazhin SS. Advanced models of fuel droplet heating and evaporation, Progress in Energy and Combustion Sci. Vol. 32, 2006, pp. 162-214.

[5] Versteeg HK, Melalasekera W. An Introduction to Computational Fluid Dynamics, Longman, 1999.

[6] Date AW. An Introduction to Computational Fluid Dynamics, Cambridge University Press, 2005.

[7] Batchelor GK. An Introduction to Fluid Dynamics, Cambridge University Press, 1967.

[8] Saffman PG. Vortex Dynamics, Cambridge University Press. 1992.

[9] Lim T, Nickels T. Vortex rings. In Fluid Vortices (ed. S. I. Green) Kluwer, Dordrecht, 1995.

[10] Kaplanski F, Sazhin SS, Fukumoto Y, Begg S, Heikal M. A generalised vortex ring model, J Fluid Mechanics, Vol. 622, 2009, pp. 233-258. 
[11] Iyer VA, Abraham J, Magi V. Exploring injected droplet size effects on steady liquid penetration in a Diesel spray with a two-fluid model, Int. J. Heat and Mass Transfer, Vol. 45, 2002, pp. 519-531.

[12] Sazhina EM, Sazhin SS, Heikal MR, Babushok VI, Johns RA. A detailed modelling of the spray ignition process in Diesel engines, Combustion Science and Technology, Vol. 160, 2000, pp. 317-344.

[13] Nijdam JJ, Guo B, Fletcher DF, Langrish TAG. Lagrangian and Eulerian models for simulating turbulent dispersion and coalescence of droplets within a spray, Applied Mathematical Modelling, Vol. 30, 2006, pp.1196-1211.

[14] Amsden AA, O'Rourke PJ, Butler TD. KIVA-II: A computer program for chemically reactive flows with sprays, Los-Alamos National Laboratory Report No. LA-11560-MS, 1989.

[15] Reitz RD, Rutland CJ. Development and testing of diesel engine CFD models, Progress in Energy and Combustion Science, Vol. 21, 1995, pp.173-196.

[16] Tonini S, Gavaises M, Theodorakos A. Modelling of high-pressure dense diesel sprays with adaptive local grid refinement, International J Heat and Fluid Flow, Vol. 29, 2008, pp. 427-448.

[17] Nurick WH. Orifice cavitation and its effect on spray mixing, Journal of Fluids Engineering, Vol. 98, 1976, pp. 681-687.

[18] Stiesch G. Modelling Engine Sprays and Combustion Processes, Springer, Berlin, London, UK, 2003.

[19] Gorokhovski MA, Saveliev VL. Analysis of Kolmogorov's model of breakup and its application into Lagrangian computation of liquid sprays under air-blast atomisation, Physics of Fluids, Vol. 15, 2003, pp.184-192.

[20] Rimbert N, Sero-Guillaume O. Log-stable laws as asymptotic solutions to a fragmentation equation: application to the distribution of droplets in a high Weber-number spray, Physical Review E, Vol. 69, 2004, paper 056316.

[21] Gorokhovski MA, Saveliev VL. Statistical universalities in fragmentation under scaling symmetry with a constant frequency of fragmentation, J. of Physics D: Appl. Phys, Vol. 41, 2008, paper 085405 .

[22] Gorokhovski MA, Jouanguy J, Chtab A. Stochastic simulation of the liquid jet break-up in a high-speed air co-flow. International Conference on Multiphase Flow, ICMF 2007, Leiptzig, July 9-13, 2007, paper S4_Tue_B_17, Book of Abstracts, p. 243.

[23] Gorokhovski MA, Herrmann M. Modelling primary atomisation, Ann Rev. Fluid Mech, Vol. 40, 2008, pp. 343-366.

[24] Sazhin SS, Martynov SB, Kristyadi T, Crua C, Heikal MR. Diesel fuel spray penetration, heating, evaporation and ignition: modelling versus experimentation, International $J$ of Engineering Systems Modelling and Simulation, Vol. 1, 2008, pp. 1-19.

[25] Reitz RD, Modelling atomization processes in high-pressure vaporizing sprays, Atomisation and Spray Technology, Vol. 3, 1987, pp. 309-337.

[26] Patterson MA, Reitz, RD. Modelling the effects of fuel spray characteristics on Diesel engine combustion and emission, SAE paper 980131, 1998.

[27] Karimi K, Sazhina EM, Abdelghaffar WA, Crua C, Heikal MR, Gold MR. Development in diesel spray characterisation and modelling, In: Proceedings of THIESEL-2006, Valencia, Spain, 2006.

[28] Sazhin SS, Krutitskii PA, Martynov SB, Mason D, Heikal MR, Sazhina EM. Transient heating of a semitransparent spherical body, International J Thermal Science, Vol. 46, 2007, pp. 444-457.

[29] Abramzon B, Sirignano WA. Droplet vaporization model for spray combustion calculations, International J Heat and Mass Transfer, Vol. 32, 1989, pp.1605-1618. 
[30] Sazhin SS, Kristyadi T, Abdelghaffar WA, Heikal MR. Models for fuel droplet heating and evaporation: comparative analysis, Fuel, Vol. 85, 2006, pp. 1613-1630.

[31] Maqua C, Castanet G, Grisch F, Lemoine F, Kristyadi T, Sazhin SS. Monodisperse droplet heating and evaporation: experimental study and modelling, International $J$ of Heat and Mass Transfer, Vol. 51, 2008, pp. 3932-3945.

[32] Abramzon B, Sazhin S. Droplet vaporization model in the presence of thermal radiation, International J Heat Mass Transfer, Vol. 48, 2005, pp. 1868-1873.

[33] Abramzon B, Sazhin S. Convective vaporization of fuel droplets with thermal radiation absorption, Fuel, Vol. 85, 2006, pp. 32-46.

[34] Kryukov AP, Levashov VYu., Sazhin SS. Evaporation of diesel fuel droplets: kinetic versus hydrodynamic models, International J Heat Mass Transfer, Vol. 47, 2004, pp. 25412549.

[35] Shishkova IN, Sazhin SS. A numerical algorithm for kinetic modelling of evaporation processes, J Computational Physics, Vol. 218, 2006, pp. 635-653.

[36] Sazhin SS, Shishkova IN, Kryukov AP, Levashov VYu., Heikal MR. Evaporation of droplets into a background gas: kinetic modelling, International J Heat Mass Transfer, Vol. 50, 2007, pp. 2675-2691.

[37] Sazhin SS, Shishkova IN. A kinetic algorithm for modelling the droplet evaporation process in the presence of heat flux and background gas, Atomization and Sprays, 2009 (in press).

[38] Tsuruta T, Nagayama G. 2004, Molecular dynamics studies on the condensation coefficient of water, J Phys Chem, Vol. B108, 2004, pp.1736-1743.

[39] Maruyama S. Molecular dynamics method for micro/nano systems, In 'Handbook of Numerical Heat Transfer', edited by W.J. Minkowycz, E.M. Sparrow and J.Y. Murthy, Chapter 21, John Wiley \& Sons, 2006.

[40] Cao B-Y, Chen M, Guo ZY. Temperature dependence of the tangential momentum accommodation coefficient for gases, Applied Physics Letters, Vol. 86, 2005, 091905 (SCI:924PT, EI:05179056781, Inspec: 8403277, IF: 3.977).

[41] Sazhin SS, Shishkova IN, Kryukov AP, Levashov VYu., Heikal MR. Evaporation of droplets into a background gas in the presence of heat flux: kinetic and hydrodynamic modelling. Proceedings of the 19th International Symposium on Transport Phenomena, 1720 August, 2008, Reykjavik, Iceland, 2008, paper 48.

[42] Sazhin SS, Shishkova IN, Kryukov AP, Levashov VYu., Heikal MR. A simple algorithm for kinetic modelling of Diesel fuel droplet evaporation. Proceedings of 6th ICCHMT May 18-21, 2009, Guangzhou, China, 2009, paper 151 (in press). 